\title{
Endoscopic discectomy as an effective treatment of a herniated intervertebral disc
}

\author{
Mlaka $\mathrm{J}^{1}$, Rapcan $\mathrm{R}^{2}$, Burianek $\mathrm{M}^{3}$, Rapcanova $\mathrm{S}^{2}$, Gajdos $\mathrm{M}^{4}$, Kocanova $\mathrm{H}^{5}$, Griger $\mathrm{M}^{6}$, \\ Kovalicova $\mathrm{L}^{2}$, Vaskova $\mathrm{J}^{7}$, Kocan $\mathrm{L}^{8}$
}

EuroPainClinics, Kosice, Slovakia. kocanladislav@gmail.com

\begin{abstract}
OBJECTIVE: Prospective observational multicentre two-arm parallel study describing clinical outcome after endoscopic discectomy provided via transforaminal and interlaminar approach.

BACKGROUND: Endoscopic lumbar discectomy (ELD) is a percutaneous minimally invasive procedure for the treatment of herniated lumbar discs. Herniations at lumbar intervertebral disc levels of L1/2, L2/3, L3/4 and $L 4 / 5$ are mostly accessed by the transforaminal (TF) approach. However, due to the anatomic position of the iliac crest, the L5/S1 level might not be reachable by the transforaminal approach, while the interlaminar (IL) percutaneous approach should be a suitable alternative.

METHODS: In a prospective observational multicentre clinical trial NCT0274311, we compared the clinical outcomes of two groups of patients who underwent ELD via IL (83) and TF (103) approach. The subjects were followed for 12 months via planned examinations by pain physicians. The levels of leg pain and back pain intensity were assessed by an 11-point numerical ratings scale (NRS). Patient's functional disability was assessed by the Oswestry Disability Index (ODI).

RESULTS: Study subjects showed a significant decrease in ODI scores in both groups $(p<0.001)$ The values of mean preoperative ODI in TF and IL groups were $39.1 \pm 15.7$ and $43.4 \pm 16$, respectively. Postoperative values in the latter groups were $14.8 \pm 14.9$ and $17.5 \pm 14.3$, respectively. Significantly lower pain scores for leg pain $(p<0.001)$ and back pain $(p<0.001)$ were also recorded at 12 -month follow-ups.

CONCLUSION: Because both procedures are strictly percutaneous; they are now more commonly performed by interventional pain physicians as a safe and effective alternative to open surgical spine procedures (Tab. 3, Fig. 7, Ref. 19). Text in PDF www.elis.sk.

KEY WORDS: endoscopic discectomy, disc herniation, transforaminal, interlaminar, pain, quality of life, radicular syndrome.
\end{abstract}

\section{Introduction}

Lumbosacral radicular syndrome is the most common cause of neuropathic pain in our population (1). The most common reason for the development of acute lumbosacral radicular syndrome is the herniation of an intervertebral disc. The peripheral nerve damage leads to significant cellular and molecular changes not only at the level of axon, but also at that of DRG. The inflammatory cascade is set off directly by the nerve damage, as well as due to the pres-

\footnotetext{
${ }^{1}$ EuroPainClinics, Kosice, Slovakia, ${ }^{2}$ EuroPainClinics, Bardejov, Slovakia, ${ }^{3}$ EuroPainClinics, Prague, Czech Republic, ${ }^{4}$ Louis Pasteur University Hospital, Neurosurgery Clinic, Kosice, Slovakia, ${ }^{5} \mathrm{Clinic}$ of Anaesthesiology and Intensive Care Medicine, Railway Hospital and Clinic Kosice, Slovakia, ${ }^{6}$ EuroPainClinics, Bratislava, Slovakia, ${ }^{7}$ Department of Medical and Clinical Biochemistry, Faculty of Medicine, PJ Safarikiensis University, Kosice, Slovakia, and ${ }^{8} \mathrm{Clinic}$ of Anaesthesiology and Intensive Care Medicine, East Slovak Institute of Cardiovascular Disease, Kosice, Slovakia

Address for correspondence: $\mathrm{L}$. Kocan, MD, PhD, Clinic of Anaesthesiology and Intensive Care Medicine, East Slovak Institute of Cardiovascular Disease, Ondavská 8, 04011 Kosice, Slovakia

Phone: +421557891100
}

ence of highly inflammatory material from the nucleus of the disc. Inflammation induces the production of neurotrophins and changes the neuronal transmission. In the place of the damaged axons and mainly in the corresponding DRG, ectopic discharges are fired, which explains the formation of radicular pain (2).

The mechanisms of central sensitisation often propagate pain to the neighbouring spinal segments. That is why, if there is a pathology seen in numerous segments on an MRI, it is necessary to perfectly correlate the MRI results with the clinical signs. When in doubt which root is causing the symptoms, it is possible to use a diagnostic root block in conjunction with physical examination and MRI (3).

There is no clear evidence that an early surgical decompression of a herniated disc has a better long-term outcome than conservative therapy. Moreover, there are a large number of opinions about how soon one should perform the surgical procedure (4).

In general, an early intervention is indicated in patients with the extinction phenomenon - sensory or motor. In patients without extinction phenomena and only with radicular pain, an early surgical decompression enables a significantly quicker return to a normal pain-free life. In the past couple of years, minimally in- 


\section{9-205}

vasive procedures have been coming forward in the field of surgical interventions. Endoscopic discectomy is among the most sparing procedures, which is performed by a spinal surgeon or an interventional pain physician.

In contrast to an open surgery, an endoscopic discectomy causes less trauma to the surrounding tissues, there is minimal blood loss and a decreased risk of post-operative fibrosis. Longterm results are, however, equivalent to an open surgery approach (5). The procedure only requires hospitalisation of the patient for one day, after which the patient leaves for home.

\section{Materials and methods}

\section{Ethics}

Ethical approval for this study (Ethical Committee No EK:9N-2015) was provided by the Ethical Committee of Medical faculty, University of PJ Safarikiensis in Kosice. The study was registered in ClinicalTrials.gov (NCT02742311). All patients gave their consent after receiving extensive information about the study.

\section{Study design}

The study is a prospective observational multicentre two-arm parallel study of endoscopic discectomy provided in acute sciatica caused by a herniated intervertebral disc. We independently investigated the efficacy of two surgical approaches: transforaminal and interlaminar approach. Included pain clinics with recruitment and postoperative follow-up were situated in Bratislava, Kosice and Bardejov. The investigation of clinical parameters was based on a numerating rating scale of pain (NRS), Oswestry disability index (ODI), patient status, patient satisfaction score and EQ-VAS. We collected all data before the procedure and 12 months after the procedure. All patients had their unique ID code generated after being enrolled in the study. To secure the safety of data, they were encrypted in an established online database. An independent study coordinator from the East Institute of Cardiovascular Diseases was appointed to superintend during the study process.

\section{Patients}

Patients classified by the American Society of Anesthesiologists physical status (ASA PS) classes I, II, or III aged between 18-75 with acute sciatica, scheduled for endoscopic discectomy after obtaining their informed consent were enrolled into the study.

Indication criteria included unilateral lumbosacral radicular pain lasting less than one year, with or without signs of radiculopathy, correlated with isolated single level disc herniation on MRI, responding neither to conservative therapy nor to transforaminal steroids 2 weeks after injection.

Exclusion criteria included multisegmental or bilateral pain, herniations in multiple adjacent segments, severe disc degeneration, radiological or clinical signs of segmental instability, pathology not suitable for endoscopic treatment e.g. bulging disc, severe MODIC changes in adjacent endplates or very large sequesters.

The study was aimed at performing a parallel statistical comparison of two endoscopic approaches, namely transforaminal (103 patients) and interlaminar (83 patients) ways of treatment of lesions in the epidural space at the level of lumbar and sacral spine segments examined before the procedure and after 12 months (Fig. 1).

\section{Surgical procedure}

\section{Transforaminal endoscopic discectomy (TFED)}

The procedure is done under monitored anaesthesia care with the patient in a prone position. After aseptic preparation of the operation field, a local infiltration of skin and muscle with trimecaine was performed. At a site located 10 to $14 \mathrm{~cm}$ from the midline, a skin incision was made, and under fluoroscopic control, a needle and a guide-wire were inserted to the level of the upper third of the superior articular process (SAP) of the affected spine segment. After puncture channel dilation, a Tom Shidi needle was delicately hammered through the SAP not exceeding the medial pedicular line. A part of SAP was removed with a series of bone drills. After inserting the endoscope, the remaining part of the ligamentum flavum was removed. The nerve root and disc material were identified

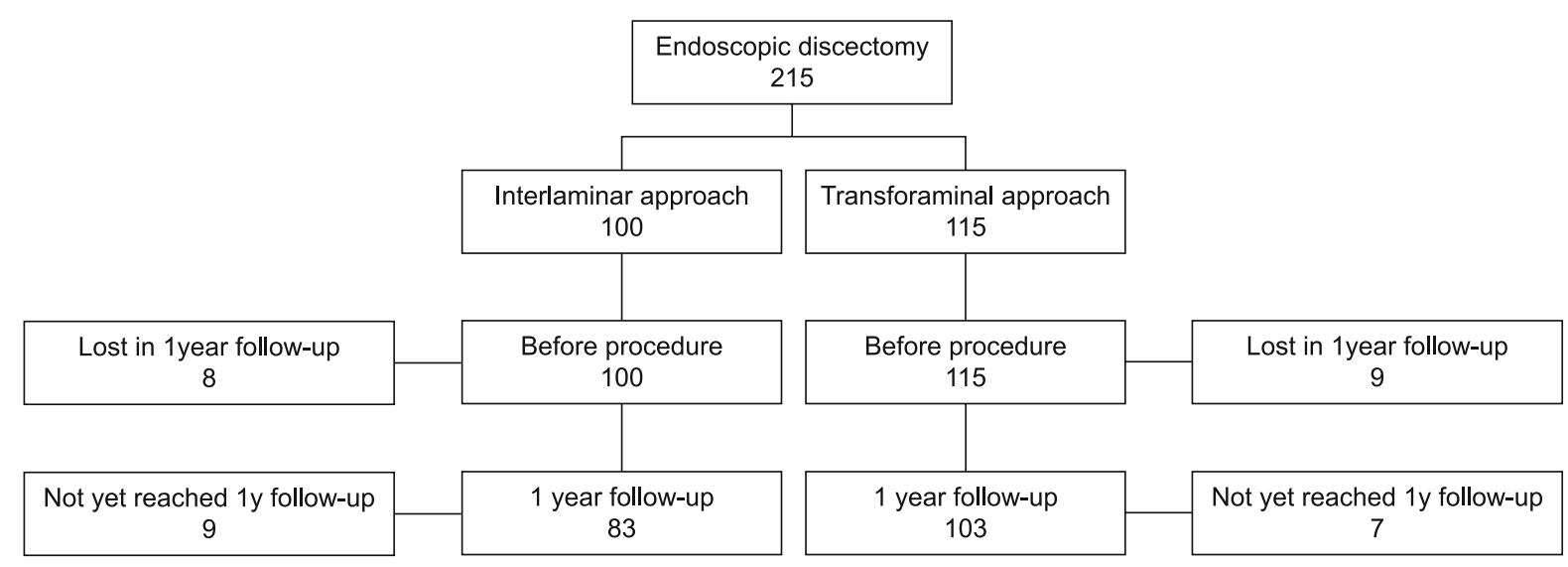

Fig. 1. Flow chart of patient selection, enrolment, and follow-up in the study. 


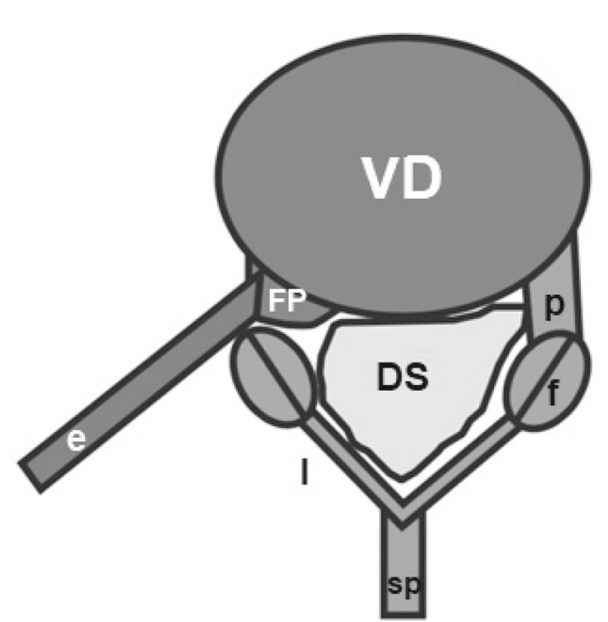

Fig. 2. Endoscopic transforaminal approach - axial view: VD - vertebral disc; FP - foraminal protrusion; I - lamina; $\mathbf{p}$ - pedicle; sp - spinous process; $f$ - facet joint; DS - dural sac; $e$ - endoscope.

and the nerve was mobilised with a flexible probe. The protrusion was removed with a grasper. The posterior annulus radiofrequency shrinkage and coagulation of epidural veins was performed with the Elliquence TriggerFlex probe. After the free pulsation of the traversing nerve root had been observed, the endoscope was removed and skin sutures applied.

Foraminal and paramedial hernias were surgically treated through a foraminal approach.

This technique uses a natural entrance into the spinal canal (neuroforamen) (Fig. 2).

The working channel for the endoscope is inserted lateromedially through the paravertebral musculature. During TFED, it is not necessary to disrupt the dorsal border of the spinal canal (skeletal lamina of the vertebra and ligamentum flavum). The risk of post-operative fibrosis formation is, therefore, minimal.

\section{Interlaminar endoscopic discectomy (ILED)}

The procedure is done under general anaesthesia care with the patient in a prone position. After aseptic preparation of the operation field, local infiltration of skin and muscle with trimecaine was performed (6). The puncture point for the guiding tubus at the affected disc level was established as a site located laterally from the spinous process, medio-caudally from the upper lamina, and over the epidural window. Subsequently, in an AP view, the guiding tubus was inserted towards the epidural window on the affected side. The insertion of the tubus was gradually checked in an AP lateral view until contact with the vertebral lamina. A working channel for the endoscope was established. Under endoscopic control, the ligamentum flavum was dissected to enter the epidural space. The nerve root, dura and disc protrusion were identified. The protrusion was removed with a grasper. Posterior annulus radiofrequency shrinkage and coagulation of epidural veins was performed with an Elliquence TriggerFlex probe. After the free pulsation of the traversing nerve root had been observed, the endoscope was removed and skin sutures applied.
When it was technically more difficult to reach central protrusions or in case of more voluminous sequesters especially at the L5/S1 level, the interlaminar approach was used (Fig. 3). A working channel with an endoscope was introduced in the midline, very similar to microdiscectomy. To enter the spinal canal, it is only needed to disrupt a part of the ligamentum flavum. The dural sac is then moved medially with a special manoeuvre to enable work in the central part of the channel.

\section{Anaesthesia}

Anaesthesia was provided by an anaesthesiology team. During the procedure, the patient was analgosedated. 10ug Sufentanil and 50-400 mg Propofol was used. During the tunnelling of the working channel, the patient was under total intravenous anaesthesia with protective lung ventilation. During the procedure, basic vital functions were monitored, namely the non-invasive blood pressure monitoring, heart rate, oxygen saturation, and respiratory rate.

\section{Scoring systems}

The monitored parameters included interval scales and index scoring systems as follows: numerical pain scale of legs, numerical pain scale of the lower back, Oswestry disability index, patient status, and patient satisfaction score.

\section{Numerical pain scale}

The numerical pain scale is an internationally accepted tool to determine the intensity of pain. The patient uses an 11-point scale $(0-10)$ to describe their intensity of pain, where 0 means no pain and 10 means the strongest possible pain the patient can imagine.

\section{Oswestry disability index}

Oswestry disability index is derived from the Oswestry low back pain questionnaire which quantifies disability caused by pain

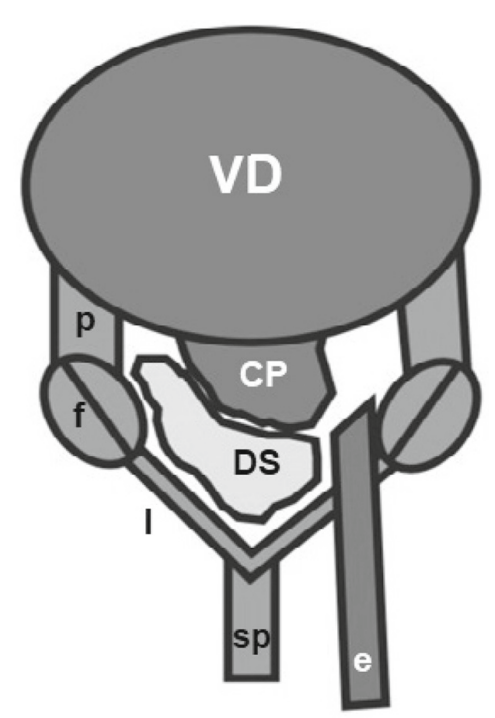

Fig. 3. Endoscopic interlaminar approach axial view: VD - vertebral disc; $\mathbf{C P}$ - central protrusion; $\mathbf{l}$ - lamina; $\mathbf{p}$ - pedicle; $\mathbf{s p}$ - spinous process; $f$ - facet joint; DS - dural sac; $e$ - endoscope. 


\section{9-205}

of the lower back. ODI is presently the gold standard method of measuring the level of disability, as well as quality of life in people suffering from lower back pain. The questionnaire includes 10 questions about the intensity of pain, lifting weights, level of selfcare, ability to walk, sit and lead a sexual life, ability to stand, have a social life, quality of sleep and ability to travel. For each question the patient can choose from 6 different answers describing various alternatives. The answers are in an order from most to least favourable. The patient chooses the answer which most closely relates to their condition. The answers are evaluated from 0 to 5 points. The most favourable answer is evaluated with 0 points (the lowest level of disability) and the last answer with 5 points (the highest level of disability). The number of points obtained from answers to each of 10 questions is summed together and multiplied by 2 . The resulting number is the ODI score.

\section{Patient status}

This is a questionnaire, which directly describes the self-sufficiency and mobility of the patient. It is graded on a scale from 0 to 5, where each number represents the patient's state as follows: 0 - pain-free, i.e. the patient leads a normal life, works, does sports, etc; 1 - the patient is able to perform basic daily activities (does not participate in sports), is employed part-time; 2 - the patient is not able to work, but can take care of himself/herself; 3 - the

Tab. 1. Characteristics of patients in groups divided according to the surgical approach.

\begin{tabular}{lcc}
\hline Characteristic & Transforaminal & Interlaminar \\
\hline Gender & M 51pt/F 52pt & M 44pt/F 39pt \\
Age & $(\min 18-\max 78)$ med 47 & $(\min 23-\max 76)$ med 43 \\
ASA & $(\min 1-\max 3)$ med 2 & $(\min 1-\max 3)$ med 2 \\
L1-L2 & 0 & 0 \\
L2-L3 & 0 & 0 \\
L3-L4 & 14 & 1 \\
L4-L5 & 52 & 5 \\
L5-S1 & 41 & 77 \\
\hline
\end{tabular}

M - male; F - female; ASA - The American Society of Anesthesiologist's physical status classification system. patient is not independent, needs help with care; 4 - the patient needs home care and is bed-ridden.

\section{Patient satisfaction score}

Patient satisfaction score (PSS) - on a scale from 0 to 10 , the patient can grade their satisfaction with the performed medical procedure and its end-result, where the score 0 means the worst satisfaction and 10 highest satisfaction.

\section{EQ VAS}

The EQ VAS is a part of EuroQol licensed questionnaire and records the respondent's self-rated health on a $20 \mathrm{~cm}$ vertical, visual analogue scale $(0-100)$ with endpoints labelled as 'the best health you can imagine' (100) and 'the worst health you can imagine'(0). This information can be used as a quantitative measure of health and quality of life as judged by the individual respondents.

\section{Statistical analysis}

The statistical test (parametric/non-parametric) was chosen based on normality and number of data.

\section{Results}

Table 1 shows the demographic characteristic for both groups. The results in Table 2 demonstrate that using either of surgical approaches led to a significant improvement in the evaluated parameters after 12 months with $\mathrm{p}<0.001$. The intensity of lower back pain was considered less intense than that of the leg pain before the procedure. There was a significant improvement in the intensity of back and leg pain after 12 months, where average values of the intensity of the pain were more-or-less the same (Fig. 4 and 5). The $95 \%$ probability of the replicability of the estimate for the given values is lower in the IL group, however, the values for both groups overlap. During the evaluation of the grade of disability according to ODI, it is also clear that after performing either of types of surgical procedure, there is a substantial improvement in the quality of life of the patient at the 12-month follow up. There are

Tab. 2. Comparison of patient pain intensity, self-content score, patient status score, Quality of life (VAS) and functional disability in both groups aftera 12-month postoperative examination period.

\begin{tabular}{|c|c|c|c|c|c|c|c|c|c|}
\hline \multirow[b]{2}{*}{ Parameters } & \multirow[b]{2}{*}{ Groups } & \multicolumn{3}{|c|}{ Before procedure } & \multicolumn{3}{|c|}{12 months follow-up } & \multirow[b]{2}{*}{ CI $95 \%$} & \multirow[b]{2}{*}{$\mathrm{p}$} \\
\hline & & Mean & $\begin{array}{l}\text { St. error } \\
\text { mean }\end{array}$ & $\mathrm{SD}$ & mean & $\begin{array}{l}\text { St. error } \\
\text { mean }\end{array}$ & $\mathrm{SD}$ & & \\
\hline \multirow{2}{*}{ Oswestry disability index } & IL group & 43.36 & 1.94 & 15.98 & 14.75 & 1.24 & 14.91 & 16.288 to 26.856 & $<0.001$ \\
\hline & TF group & 39.09 & 1.71 & 14.01 & 17.52 & 1.18 & 14.31 & 16.315 to 26.83 & $<0.001$ \\
\hline \multirow{2}{*}{$\begin{array}{l}\text { Numerical pain scale } \\
\text { Back pain }\end{array}$} & IL group & 4.31 & 0.36 & 3.27 & 2.18 & 0.23 & 2.11 & 1.370 to 2.895 & $<0.001$ \\
\hline & TF group & 5.35 & 0.29 & 2.97 & 2.41 & 0.19 & 1.94 & 2.345 to 3.530 & $<0.001$ \\
\hline $\begin{array}{l}\text { Numerical pain scale } \\
\text { leg pain }\end{array}$ & TF group & 7.34 & 0.21 & 2.07 & 2.23 & 0.19 & 1.93 & 4.590 to 5.623 & $<0.001$ \\
\hline \multirow{2}{*}{ Patient status score } & IL group & 2.27 & 0.10 & 0.85 & 1.10 & 0.11 & 0.94 & 0.939 to 1.407 & $<0.001$ \\
\hline & TF group & 2.44 & 0.08 & 0.84 & 1.12 & 0.10 & 0.09 & 1.113 to 1.527 & $<0.001$ \\
\hline \multirow{2}{*}{ Patient satisfaction score } & IL group & $\mathrm{x}$ & $\mathrm{x}$ & $\mathrm{X}$ & 7.6 & 0.27 & 2.48 & 7.05 to 8.14 & $\mathrm{X}$ \\
\hline & TF group & $\mathrm{x}$ & $\mathrm{x}$ & $\mathrm{X}$ & 7.96 & 0.24 & 2.42 & 7.49 to 8.43 & $\mathrm{X}$ \\
\hline \multirow{2}{*}{ Quality of life } & IL group & 49.13 & 1.73 & 15.74 & 72.60 & 1.91 & 17.36 & -28.54 to -18.39 & $<0.001$ \\
\hline & TF group & 49.40 & 1.55 & 15.69 & 70.10 & 1.80 & 18.21 & -25.39 to -16.00 & $<0.001$ \\
\hline
\end{tabular}

$\mathrm{IL}$ - interlaminar; TL - transforaminal; St. error mean - standard error of the mean; SD - standard deviation; CI - confidence interval 
NRS - back pain

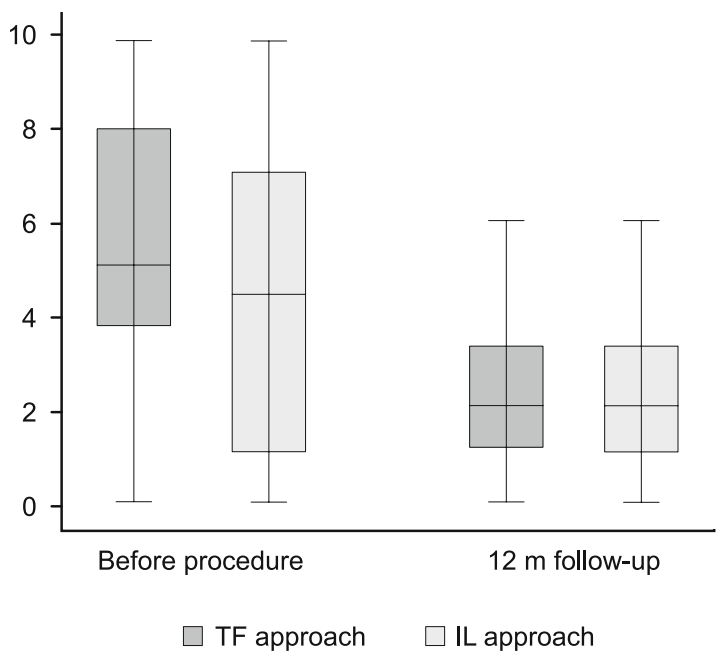

Fig. 4. Evaluation of back pain intensity in patients indicating significant reduction in low back pain in both groups after 12 months. NRS - numerating rating scale; TF - transforaminal; IL - interlaminar.

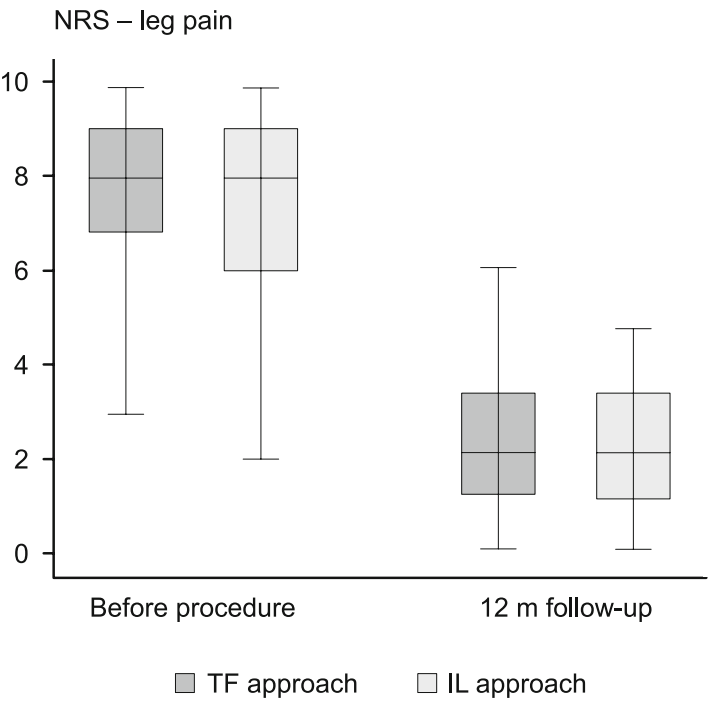

Fig. 5. Evaluation of back pain intensity in patients indicating significant reduction in leg pain in both groups after 12 months. NRS - numerating rating scale; TF - transforaminal; IL - interlaminar.

only minor differences in the CI values for both of the groups. The improvement was comparable in both groups of patients (Fig. 6).

In parallel, the evaluation of self-sufficiency and mobility of patients in the PS questionnaire demonstrated a comparable and significantly improved patient status after 12 months in both groups (Tab. 2). Their condition improved in 12 months from an average of "inability to work" to an average of "ability to take part in basic daily activities". Although, as in NRS, the CI is lower in the IL group, the ranges of both groups overlap. Considering the general patient status, the patients in both groups expressed a rather high satisfaction with the medical procedure in the PSS questionnaire,
Oswestry disability index

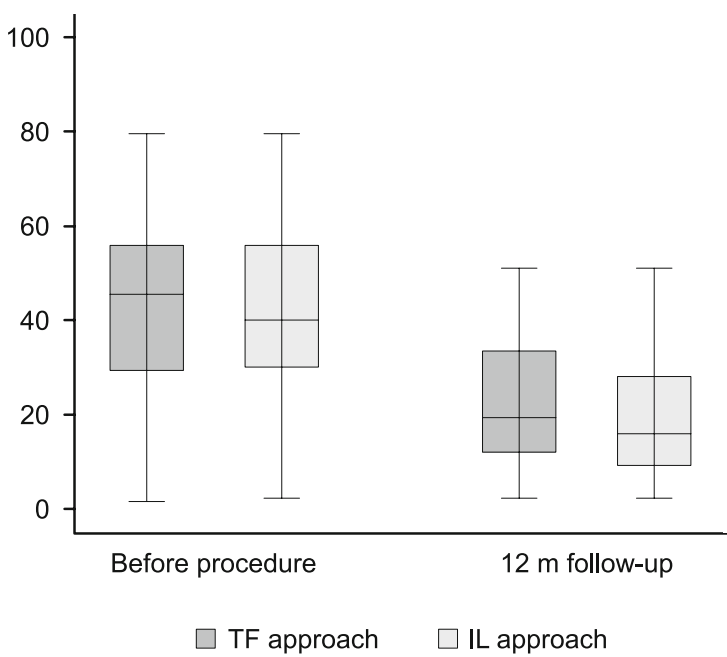

Fig. 6. Evaluation of patient functional disability by the Oswestry Disability Index indicating significant improvement in comparison with baseline after 12 months in both groups. TF - transforaminal; IL - interlaminar.

\section{Quality of life}

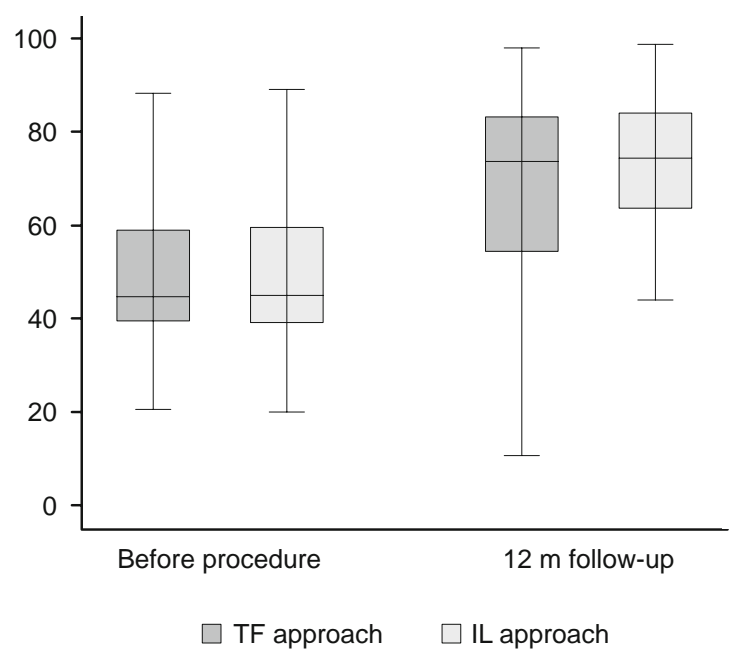

Fig. 7. Evaluation of patient quality of life measured on visual analogue scale indicating significant improvement in comparison with baseline after 12 months in both groups. TF - transforaminal; IL-interlaminar.

being mostly graded as 7 or 8 . Self-assessment of the quality of life of the patients (EQ-VAS) demonstrated a significant improvement after the procedure in both groups (Tab. 2, Fig. 7). The rate of complications is summarized in Table 3.

\section{Discussion}

The dominant position among the surgical approaches to a lumbar intervertebral disc herniation and the decompression of a spinal nerve used to belong to open surgical procedures, most commonly to hemi-laminectomy $(7,8)$. The strategy of surgical 
Tab. 3. The rate of complications.

\begin{tabular}{lcccc}
\hline Complications & TF 103pt & $\%$ & IL 83pt & $\%$ \\
\hline Nerve root injury & 0 & 0 & 0 & 0 \\
New level herniation & 3 & 2.91 & 4 & 4.82 \\
New level operation & 2 & 1.94 & 2 & 2.41 \\
Surgical errors & 0 & 0 & 0 & 0 \\
Dural puncture & 1 & 0.97 & 1 & 1.2 \\
Hematoma & 0 & 0 & 0 & 0 \\
Wound complications & 0 & 0 & 0 & 0 \\
Re-herniations & 5 & 4.85 & 4 & 4.82 \\
Reoperations & 5 & 4.85 & 4 & 4.82 \\
\hline
\end{tabular}

$\mathrm{TF}$ - transforaminal; IL - interlaminar

approaches has since gradually started focusing on the minimallyinvasive surgical options, where micro-discectomies have become the dominant choice. The trend of minimizing the surgical field in the treatment of symptomatic intervertebral disc herniations lead to the development of the endoscopic discectomy technique, (9) which has two approaches; one is interlaminar, and the other is the transforaminal approach, depending on the type of herniation and its height (10). Moreover, this surgical approach has been added to the portfolio of not only spinal surgeons, but also interventional pain management specialists. The comparison of our results between the transforaminal and interlaminar approach after a 12-month follow-up demonstrated a high effectivity of treatment in minimising back pain $\mathrm{p}<0.001$, as well as a significant decrease in the radiation of pain to the leg $p<0.001$.

The inability to carry out basic daily activities due to lower back pain is expressed by the Oswestry disability index calculated using a questionnaire based on the intensity of pain, carrying out basic daily activities, lifting bigger loads, walking, sitting, standing, sleeping, social life, sex life and traveling (11).

The character of acute pain with lumbosacral radicular syndrome is apparent by adjusting and regaining positive values in the monitored ODI score. The ODI scores and results from the patient quality of life questionnaire EuroQuol that we obtained in this study demonstrate a significant improvement after 12 months to the procedure $(p<0.001)$. The effectiveness of endoscopic discectomy procedures is comparable to an open surgery as well as to a microdiscectomy surgical approach $(12,13)$. All of these surgical approaches are of high effectiveness with excellent outcomes (5, 14). During this study, we noted a small number of complications linked to the surgical procedure. Some of the complications include nerve root injury, new level herniation, new level operation, surgical errors, dural puncture, epidural hematoma, wound complications, re-herniations and reoperations. From a meta-analysis comparing the prevalence of complications after lumbar open microdiscectomy, microendoscopic discectomy (MED) and percutaneous microdiscectomy, a smaller number of post-operative complications was found with endoscopic procedures, as well as a decrease in post-operative pain (15). This is due to minimal manipulation of the surrounding tissue. Another notable discovery in the peri-operative period was the reduction in anaesthesia-related complications. The procedure is done with the patient in a prone position, which in general causes a number of disadvantages in total anaesthesia. This is logistically the most difficult position due to the challenges associated with providing adequate oxygenation, ensuring adequate ventilation, maintaining haemodynamics, and securing intravenous lines and tracheal tube. The access to the patient's airway is also poor. Pressure sores, vascular compression or brachial plexus injuries can occur (6). However, endoscopic discectomies are performed with analgosedation combined with local anaesthesia. During the almost whole procedure, the patient communicates with the surgeon and anaesthesiologist and can notify them if they feel any unpleasant sensation or new pain associated with the medical instrument touching a spinal nerve $(16,17)$.

As compared to the open surgical approach, the advantage of a minimally invasive endoscopic discectomy lies in its radical influence on the cause of pain without further chronification, and with minimal damage it causes to the paravertebral musculature. This is confirmed upon the follow-up MRI tests which demonstrate significant atrophy of the paravertebral musculature after a microdiscectomy approach, while after an endoscopic procedure, such changes are not visualized. This phenomenon is most probably linked to the incisional denervation of muscles. Patients after endoscopic discectomy maintain their muscular corset, which enhances early rehabilitation as well as post-operation functionality $(4,18)$.

Beside the removal of the extruded fragment, the endoscopic discectomy techniques also preserve the ligamentum flavum, decrease bleeding in the epidural space and effectively resolve sciatica symptoms with a lower risk of recurrence and formation of epidural fibrosis.

In general, an early intervention is indicated in patients with the extinction phenomenon - sensory or motor. In patients without extinction phenomena and with radicular pain only, an early surgical decompression enables a significantly quicker return to their normal pain-free life $(3,19)$.

\section{Conclusion}

Endoscopic discectomy belongs to the keyhole techniques. It has become a domain not only for spinal surgeons, but also for interventional pain physicians. It presents a valuable surgical approach due to its cautiousness around surrounding tissues; it demonstrates a significant reduction in post-operative complications linked with the formation of epidural fibrosis, not to forget the benefit of early convalescence, additional decreased costs of hospitalisation, as well as decreased risk of pain becoming chronic (10). After the procedure, the patient is able to walk out of the operation theatre on his own, and, while adhering to a strict regime, can recover at home.

\section{References}

1. Van Boxem K, Cheng J, Patijn J, Van Kleef M et al. Lumbosacral Radicular Pain. Evidence-Based Interventional Pain Medicine: According to Clinical Diagnoses 2011; 10 (4): 71-86.

2. Van Boxem K, Huntoon M, Van Zundert J, Patijn J, Van Kleef M, Joosten EA. Pulsed radiofrequency: a review of the basic science as applied to the pathophysiology of radicular pain: a call for clinical translation. Regional Anesthesia and Pain Medicine. 2014; 39 (2): 149-159. 
3. Manchikanti L, Buenaventura RM, Manchikanti KN et al. Effectiveness of therapeutic lumbar transforaminal epidural steroid injections in managing lumbar spinal pain. Pain Physician 2012; 15 (3): 199-245.

4. Weinstein JN, Lurie JD, Tosteson TR et al. Surgical vs nonoperative treatment for lumbar disk herniation: the Spine Patient Outcomes Research Trial (SPORT) observational cohort. JAMA 2006; 296 (20): 2451-2459.

5. Cong L, Zhu Y, Tu G. A meta-analysis of endoscopic discectomy versus open discectomy for symptomatic lumbar disk herniation. Eur Spine J 2016; 25 (1): 134-143.

6. Rozet I, Vavilala MS. Risks and benefits of patient positioning during neurosurgical care. Anesthesiol Clin 2007; 25 (3): 631-53.

7. Munting E, Röder C, Sobottke R, Dietrich D, Aghayev E. Patient outcomes after laminotomy, hemilaminectomy, laminectomy and laminectomy with instrumented fusion for spinal canal stenosis: a propensity score-based study from the Spine. Tango registry Eur Spine J 2015; 24 (2): 358-368.

8. Oktem IS, Akdemir H, Kurtsoy A, Koc RK, Menku A, Tucer B. Hemilaminectomy for the removal of the spinal lesions. Spinal Cord 2000; 38 (2): 92-96.

9. Seiger A, Gadjradj PS, Harhangi BS et al. PTED study: design of a non-inferiority, randomised controlled trial to compare the effectiveness and cost-effectiveness of percutaneous transforaminal endoscopic discectomy (PTED) versus open microdiscectomy for patients with a symptomatic lumbar disc herniation. BMJ Open 2017; 21; 7 (12): e018230.

10. Li ZZ, Hou SX, Shang WL, Song KR, Zhao HL. The strategy and early clinical outcome of full-endoscopic L5/S1 discectomy through interlaminar approach. Clin Neurol Neurosurg 2015; 133: 40-45.

11. Khan I, Bydon M, Archer KR et al. Impact of Occupational Characteristics on Return to Work for Employed Patients after Elective Lumbar Spine Surgery. Spine J 2019; 9: S1529-9430.
12. Choi KC, Shim HK, Kim JS et al. Cost-effectiveness of microdiscectomy versus endoscopic discectomy for lumbar disc herniation. Spine J 2019; 19 (7): 1162-1169.

13. Liu X, Yuan S, Tian Y, Wang L, Gong L, Zheng Y, Li J. Comparison of percutaneous endoscopic transforaminal discectomy, microendoscopic discectomy, and microdiscectomy for symptomatic lumbar disc herniation: minimum 2-year follow-up results. J Neurosurg Spine 2018; 28 (3): 317-325.

14. Lee SH, Chung SE, Ahn Y, Kim TH, Park JY, Shin SW. Comparative radiologic evaluation of percutaneous endoscopic lumbar discectomy and open microdiscectomy: a matched cohort analysis. Mount Sinai J Med 2006; 73 (5): 795-801.

15. Shriver MF, Xie JJ, Tye EY, Rosenbaum BP, Kshettry VR, Benzel EC, Mroz TE. Lumbar microdiscectomy complication rates: a systematic review and meta-analysis. Neurosurg Focus 2015; 39 (4).

16. Henmi T, Terai T, Hibino $\mathbf{N}$ et al. Percutaneous endoscopic lumbar discectomy utilizing ventral epiduroscopic observation technique and foraminoplasty for transligamentous extruded nucleus pulposus: technical note. J Neurosurg Spine 2016; 24 (2): 275-280.

17. Zhu YJ, Ying GY, Chen AQ et al. Minimally invasive removal of lumbar intradural extramedullary lesions using the interlaminar approach. Neurosurg Focus. 2015; 39 (2).

18. Lequin MB, Verbaan D, Jacobs WC et al. Surgery versus prolonged conservative treatment for sciatica: 5-year results of a randomised controlled trial. BMJ Open 2013; $28: 3$ (5).

19. Vialle LR, Vialle EN, Henao Suárez JE, Giraldo G. Lumbar disc herniation. Revista Brasileira de Ortopedia 2010; 45 (1): 17-22.

Received October 24, 2019. Accepted December 5, 2019. 